\title{
PHIL Infrastructure in CoSES Microgrid Lab
}

This paper was downloaded from TechRxiv (https://www.techrxiv.org).

\section{LICENSE}

CC BY-NC-SA 4.0

SUBMISSION DATE / POSTED DATE

23-11-2021 / 29-11-2021

CITATION

Mohapatra, Anurag; Peric, Vedran S.; Hamacher, Thomas (2021): PHIL Infrastructure in CoSES Microgrid Lab. TechRxiv. Preprint. https://doi.org/10.36227/techrxiv.17065184.v1

DOI 


\section{PHIL Infrastructure in CoSES Microgrid Lab}

\author{
Anurag Mohapatra \\ Technical University of Munich \\ Munich, Germany \\ anurag.mohapatra@tum.de
}

\author{
Thomas Hamacher \\ Technical University of Munich \\ Munich, Germany \\ thomas.hamacher@tum.de
}

\author{
Vedran S. Perić \\ Technical University of Munich \\ Munich, Germany \\ vedran.peric@tum.de
}

\begin{abstract}
This paper describes the Power hardware-in-theloop (PHIL) architecture and capacities of the CoSES laboratory at TU Munich. The lab brings together renewable resources, flexible grid topologies, fully controllable prosumer emulators, a real-time control environment, and an API access for external connection to the lab. The electrical and control design of the lab allows for sophisticated PHIL experiments with an user-friendly implementation. Two experiments are included, to validate the PHIL performance and demonstrate the use of PHIL infrastructure to investigate an OPF algorithm.
\end{abstract}

Index Terms-Smart energy systems, Microgrid, Smart grid, Power hardware-in-the-loop, Laboratory infrastructure

\section{INTRODUCTION}

Microgrids at low voltage (LV) level featuring fully controllable prosumers connected to renewable energy sources, have emerged as key players in energy sector transition pathways. Microgrid philosophies predominantly rely on flexibility and load shifting capacity offered through sector coupling with heat and transportation networks. Furthermore, a high penetration of Information and Communication Technology (ICT) and decentralised control strategy in power systems are needed to strengthen the adoption of renewable microgrids in a wide scale. Microgrid research, therefore, focuses on small-scale, reliable multi-energy systems with limited dependence on the wide area synchronous grids through high concentration of local renewable energy resources.

Research into multi-energy microgrids with prosumer capabilities requires interdisciplinary approaches over traditional electrical power systems fields. This gap extends to a lack of laboratory and demonstration facilities for multi-energy microgrids. The Center for Combined Smart Energy Systems (CoSES) at Technical University of Munich (TUM) was established to address this gap by providing capability to emulate a small multi-energy microgrid with fully controllable electrical, heating and transportation network. The overall concept of the lab was introduced to the research community in [1].

In this paper, we present the capabilities of the laboratory electrical grid, the Power Hardware-in-the-Loop (PHIL) test beds and the associated control architecture of CoSES in detail. Two experiments are used to demonstrate the operating philosophy and research directions of the laboratory.

The construction of the CoSES laboratory was supported by Deutsche Forschungsgemeinschaft (DFG) through the project "Flexible reconfigurable microgrid laboraotry" under Project number 350746631. The work of Vedran S. Perić was supported by DFG through the project "Optimal Operation of Integrated Low-Temperature Birdirectional Heat and Electric Grids (IntElHeat)" under Project number 450821044.

\section{OVERVIEW OF COSES ELECTRICAL GRID}

\section{A. Philosophy}

The electrical grid design of CoSES is based on the following key features,

1) Emulate and not simulate: Use of Real-Time (RT) simulators for detailed grid models, requiring validation, is the dominant trend in power systems laboratories. CoSES uses an actual grid, prosumers and Distributed energy resources (DER), wherever possible. Concepts which cannot be represented with the real hardware are emulated with test beds to mimic the dynamic response.

2) Flexible grid configuration: A laboratory for microgrid experiments should possess the following properties: 1) switching between radial or meshed topology, 2) flexibility in cable lengths, 3) re-ordering of buses, 4) flexibility of prosumer location, 5) variable DER capacities, and 6) grid or islanded mode.

3) User friendliness in changing configurations: The flexibility in grid arrangement must be easy to implement for less delay in between measurements. Experiment specific configurations like, the location of prosumers, DERs and grid topology must be electrically safe to change for a researcher without LV electrician qualifications.

4) Minimize power losses for full load experiments: A microgrid can be rated at more than $100 \mathrm{kVA}$ and such power cannot be realistically consumed in a laboratory for long duration. Therefore, to conduct continuous PHIL experiments at rated current and voltages, a circulation loop into the supply grid must be established through the lab.

\section{B. Grid}

The CoSES electrical system is described in the Fig. 1 Two tap changing transformers connect a LV cable network, spread over a maximum of 10 buses, to the Munich city grid. The power cables have a total length of approximately $1.8 \mathrm{~km}$ and are distributed over 12 cable sections which can be connected between two arbitrary LV buses. The four core NYCWY cables of $70 \mathrm{~mm}^{2}, 95 \mathrm{~mm}^{2}$, and $150 \mathrm{~mm}^{2}$ diameter are available. The currently used segments are mentioned in blue in the Fig. 11 The grid topology can be altered by changing the cable lengths between two buses. Radial and meshed topologies can be accomplished using the circuit breaker labeled CB2 in Fig. 1 If needed, the grid can also be split into two separate radial distribution grids by opening 


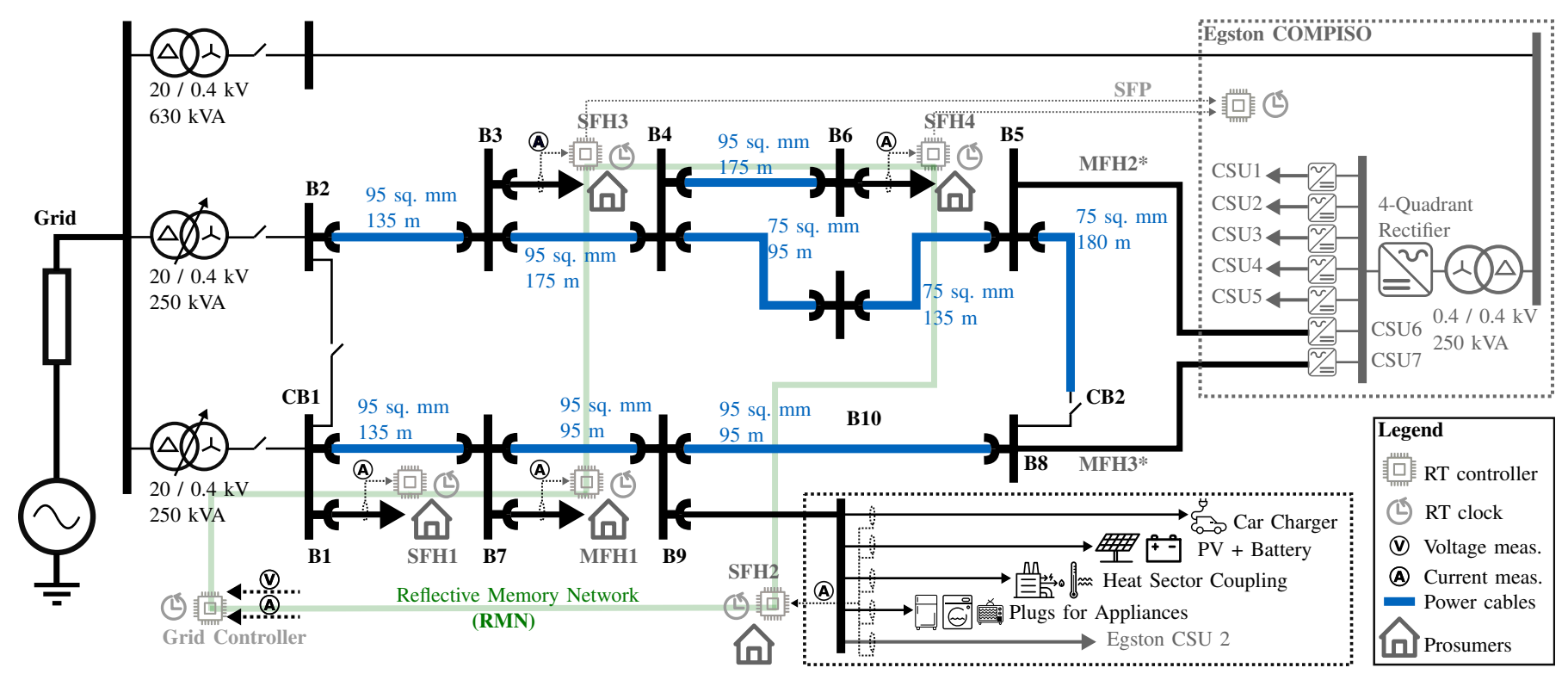

Fig. 1. CoSES PHIL grid schematic

$\mathrm{CB} 1$ and $\mathrm{CB} 2$ in Fig. 1. A separate $630 \mathrm{kVA}$ transformer is used to provide a current feedback path for PHIL experiments. This allows the lab to perform experiments at the rated power levels of a LV distribution grid, while consuming only the power equivalent of the losses on the lines.

As mentioned in [1], the lab is designed as 4 single family houses (SFH) and one multi family house (MFH) connected together in a distribution grid of flexible topology. For the electrical grid, each of these houses is realised as a LV distribution board which can be connected to any of the 10 LV buses using short CEE [2] connectors, as seen in Fig. 2. This affords great ease and flexibility in moving the prosumer nodes across the grid for scenario studies within the same experiment context.

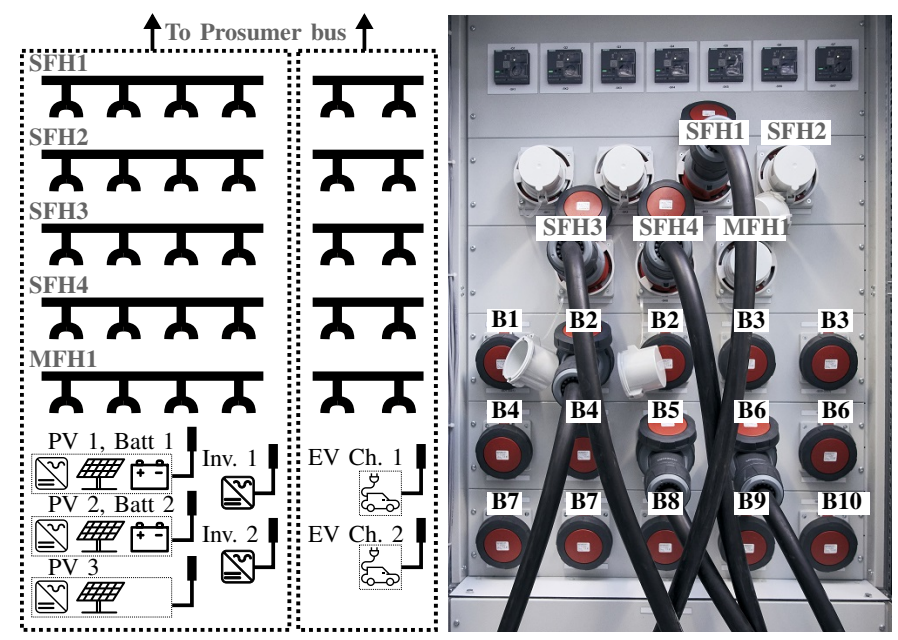

Fig. 2. DER (left) and Houses (right) connection cabinet

\section{Prosumer buses}

CoSES offers five prosumer nodes, represented as houses, and each of them has access to PV, battery storage, electric car chargers, prosumer emulators and a coupling to the heat network. They are labelled as Single family house (SFH) or Multi family house (MFH) and an inset image of the prosumer bus for SFH2 is shown in Fig. 1 .

1) Prosumer emulators: The Egston COMPISO System Unit (CSU) works as the PHIL emulator in CoSES [3]. The Egston CSUs are series of seven 4-leg inverters which share the same DC bus and can emulate bi-directional prosumer behaviour. The inverters can individually generate or consume upto $100 \mathrm{kVA}$ at LV grid voltage, and have a combined load capacity of $230 \mathrm{kVA}$ taken over all seven inverters. They can inject harmonics up to $5 \mathrm{kHz}$ and can be programmed as active power filter. They can also be configured as voltage or current source inverter which allows the lab to either operate in island or grid connected mode. Five CSU inverters are connected to the prosumer nodes as shown in Fig. 11. The remaining two CSUs are directly connected to the LV grid at Bus B5 and B8. These can emulate two extra prosumers in CoSES, shown as MFH2* and MFH3* in Fig. 1.

2) DER: Each prosumer node in CoSES, in addition to the Egston CSU emulators, has a possible connection to photovoltaics (PV) - 2 x $5 \mathrm{~kW} \& 1$ x $10 \mathrm{~kW}$, batteries 2 x $13 \mathrm{kWh}$, electric vehicle (EV) chargers - 2 x $22 \mathrm{~kW}$, and household plugs. These connections are shown for SFH2 Fig. 1, in To allow for maximum flexibility in connecting prosumer nodes to resources, a CEE connector switchboard design, shown in Fig. 2 is implemented for PV-battery systems and EV chargers. Thus every prosumer can have a variety of DER capacities depending on the experiment requirements. A short cable with CEE connectors at both ends can be used to move a prosumer node to any bus within the CoSES grid, as can be seen in the cabinet image of Fig. 2 The design for the DER and prosumer patch cabinet, makes it safe and user friendly to rearrange the resource within CoSES grid. 


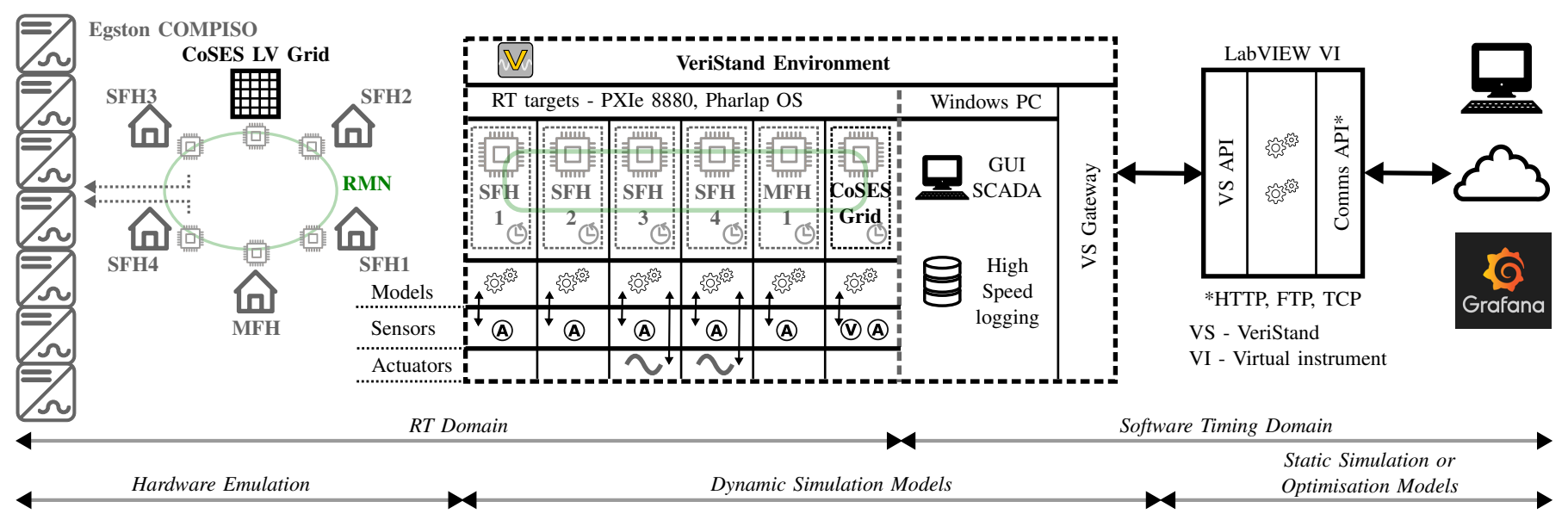

Fig. 3. CoSES control system schematic

\section{CONTROL AND INSTRUMENTATION}

\section{A. Philosophy}

Keeping with the interdisciplinary nature of multi-energy microgrid research and decentralised operation schemes, the control philosophy of CoSES is based on the following key features,

1) Free choice of toolchain: Researchers should be able to use different control design or power system simulation software, according to their individual preference, to foster co-operative work in an interdisciplinary field.

2) Multiple timescales: Within PHIL test benches, the power control and current control loops can vary a whole order of magnitude. Furthermore, static simulations and optimisation models for energy management systems must be incorporated to PHIL experiments to validate microgrid control schemes.

3) Distributed instrumentation and computing: In CoSES, microgrid operation and prosumers are monitored and controlled by a set of independent Real-Time (RT) resources, communicating with each other, as opposed to the centralised approach used in many power system test benches.

4) Open to commercial protocols: PHIL test benches for microgrid research should be open to industry standard protocols and interface with commercially available smart grid components, to ensure easy replication in real world scenarios.

The control philosophy of CoSES is represented in Fig. 3 and the individual components are explained further in subsequent sections.

\section{B. Controllers}

CoSES uses a National Instruments (NI) hardware and software ecosystem to realise the RT multi-energy grid control system environment. For the electrical PHIL experiments, each of the five prosumer nodes are provided with a PXIe8880 embedded controller for the local prosumer control and measurement tasks. An extra controller is provided for the CoSES LV grid buses to collect bus voltage and branch current measurements. The controllers run the PharLap OS and are compatible with a variety of IO cards. The locations of the controllers is shown in Fig. 1. Within CoSES, the controllers operate at either $5 \mathrm{kHz}$ or $10 \mathrm{kHz}$ RT target rate for PHIL experiments. An asynchronous communication ring link, the Reflective Memory Network (RMN), is used to transfer data from one controller to another within $1 \mathrm{~ms}$ delay, as shown in Fig. 1 and 3. This setup allows a distributed RT control scheme for PHIL experiments where grid measurements, monitoring, prosumer current injection can be separated into different RT targets.

Distributed computation resources also allow for multiple experiments to be conducted simultaneously if the PHIL configurations do not interfere with the each other. The RMN asynchronous link transfers data from RT targets which are not part of the current experiment. Thus multiple PHIL experiments can take place simultaneously by sharing the LV grid measurements over the RMN and not blocking the central grid RT controller.

Current or voltage setpoints are sent to the Egston CSUs, acting as prosumer emulators, through a SFP link Input/output (IO) card connected to the RT controllers. This card is available in SFH3 and SFH4 controllers, as seen in Fig. 1 and 3 Each card can provide setpoints to maximum four CSUs. The setpoints are transferred as sampled points to the Pulse width modulation controller of the Egston CSU. The control algorithm in the RT target converts the power setpoints to continuous waveforms sends over to the CSU at $4 \mu$ s intervals.

NI VeriStand (VS) [4], is used as the software environment for PHIL applications in CoSES and is represented in the center of Fig. 3. VS can be deployed over Windows PCs and embedded RT targets running PharLap or NI Linux RT OS. Researchers can prepare their control models using a variety of tools, not limited to, MATLAB/Simulink, LabVIEW, Dymola, $\mathrm{C}, \mathrm{C}++$ and Python. VS accepts the compiled models as .dll or Functional Mock-up Interface (FMI) file. Once compiled, the models can be assigned to any RT target within the CoSES network. VS reads and maps IO from other RT targets, included in the project, using the RMN feature, albeit at a maximum $1 \mathrm{~ms}$ delay. Models can be run at different execution rates using a decimation of the RT target rate.

The VS project is configured, deployed and monitored over 
a Windows Host PC. A rudimentary GUI can be configured as an operator control screen to interact with the PHIL experiment and log results. High speed logging upto the RT target rate is possible for electrical measurements. VS offers an API for LabVIEW, .NET and C\# to interact with the deployed VS project from external software. Although this connection is not RT, it can be used to transfer setpoints from an optimisation routine, cloud database, a co-simulation or an energy management system with commercial communication protocols. This methodology was leveraged to develop an open-source IoT platform based monitoring and logging tool [5] for CoSES.

\section{Measurements}

CoSES uses a total of 246 Hall effect and closed loop fluxgate transducers for current and voltage measurements respectively in the lab [6]. The grid RT target collects all bus voltages and branch currents, while the prosumer RT targets generate appropriate control action of DERs and the Egston CSU. Since the measured bus voltage phase and magnitude information are required for generating injection current setpoints, the measured signals are passed from the grid RT controller to the Prosumer RT target over RMN. The communication delay over RMN must be corrected to maintain synchronisation to the grid using PLL. This distributed measurement concept has been shown in [7] along with validation of the harmonic estimation and recovery methods.

Time synchronization between RT targets is integral for accurate measurements and feedback loops in PHIL test benches with distributed control schemes. The RT targets in CoSES are synchronized with the NI-6683H timing and synchronisation card. The card offers synchronization over a 1588 IEEE Precise Time Protocol switch or through an external GPS antenna. Both of these methods are available in CoSES for the PHIL experiments. The Egston CSUs carry their an on-board FPGA controller which must also be synchronized with the CoSES controllers for power injections in grid connected mode. The SFP link between CoSES controllers and the Egston CSUs strobes at $4 \mu \mathrm{s}$. This property is used to make the CSU onboard clock a slave to the SFP strobe. Thus a laboratory wide time synchronization is achieved over six RT targets and the onboard controllers of the seven CSU cabinets.

\section{EXPERIMENTS}

We present results of two typical experiments in this section to highlight the performance of CoSES PHIL setup. In the first part we demonstrate the precision and response of the Egston CSUs as PHIL agents. In the second part we conduct an online optimal power flow (OPF) based redispatching of three CSUs, while three others emulate some standard load profiles.

\section{A. PHIL validation}

The Egston CSU for SFH3 is used for the validation of the PHIL capacities in CoSES. We operate the CSU at SFH3 as a current source inverter, as shown in Fig. 11, for unbalanced three phase references. The inverter is connected at bus B3 while bus $\mathrm{B} 1$ is connected to the public grid. The grid synchronous inverter current references are generated from user defined active power setpoints, voltage measurements and PLL at Bus B3. All other prosumer nodes are disconnected for this experiment. In Fig. 4, we see the fundamental and harmonics current setpoint tracking by the Egston CSU at SFH3 for phase A. A harmonics toggle switch is used to introduce $3^{r d}$ and $5^{\text {th }}$ order components to the current injection. The amplitude of harmonics is chosen to be $12.5 \%$ of the fundamental magnitude. It can be seen that the signal reproduction is accurate and the jump from purely fundamental to fundamental with harmonics reference is near instantaneous. The total harmonic distortion (THD) and magnitude values, provided in the Tab. I. show very close reproduction of the signal through the PHIL components.

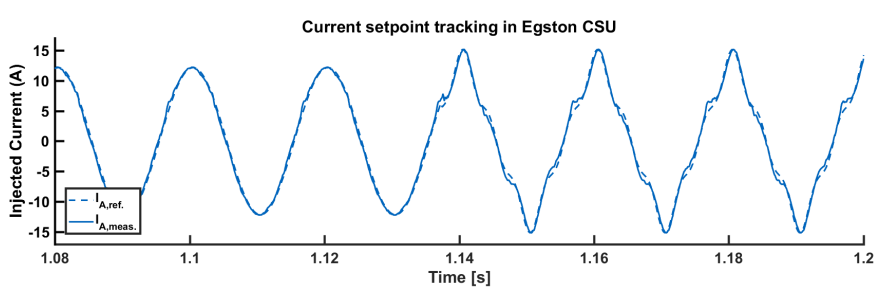

Fig. 4. PHIL validation results - harmonics and setpoint tracking

TABLE I

THD AND MAGNITUDES FOR PHIL VALIDATION

\begin{tabular}{|l|l|l|l|l|}
\cline { 2 - 5 } \multicolumn{1}{c|}{} & \multicolumn{2}{l|}{ Pure 50Hz Injection } & \multicolumn{2}{l|}{$\mathbf{5 0 H z}$ with Harmonics Injection } \\
\cline { 2 - 5 } \multicolumn{1}{c|}{} & Reference & Measured & Reference & Measured \\
\hline THD \% & 0.03 & 2.21 & 17.40 & 17.49 \\
\hline$\left|I_{50 \mathrm{~Hz}}\right|$ (A) & 12.13 & 12.14 & 12.13 & 12.14 \\
\hline$\left|I_{150 \mathrm{~Hz}}\right|$ (A) & - & - & 1.497 & 1.463 \\
\hline$\left|I_{250 \mathrm{~Hz}}\right|$ (A) & - & - & 1.488 & 1.493 \\
\hline
\end{tabular}

Fig. 5 shows the unbalanced three phase active power injection from the Egston CSU at SFH3. Phase A is held constant at a $2 \mathrm{~kW}$ generation, while the phases $\mathrm{B}$ and $\mathrm{C}$ switch between generation to load and vice-versa within the observation window to highlight a dynamic prosumer emulation. It can be see that the three phases can track their respective power setpoints independently and are suitable for unbalanced distribution grid emulation. In the bottom left of Fig. 5. a $8 \mathrm{~kW}$ power swing on phase $\mathrm{C}$, from generation to load behaviour, is achieved. The instantaneous power changes within $10 \%$ of the cycle, and the averaged power takes up to two cycles to settle to this change. The bottom right of Fig. 5. shows the change in the current waveform with respect to the bus voltage at Phase B. As the Egston CSU transitions from generation to load, we see a near instantaneous change in phase difference shift from $180^{\circ}$ to $0^{\circ}$.

\section{B. Online Optimal Power Flow (OPF) on CoSES PHIL}

In this experiment, we use six Egston CSUs - three as generators and three as loads - divided in two clusters, as seen in Fig. 6. The two clusters can be imagined as two separate microgrids, which do not freely share information 

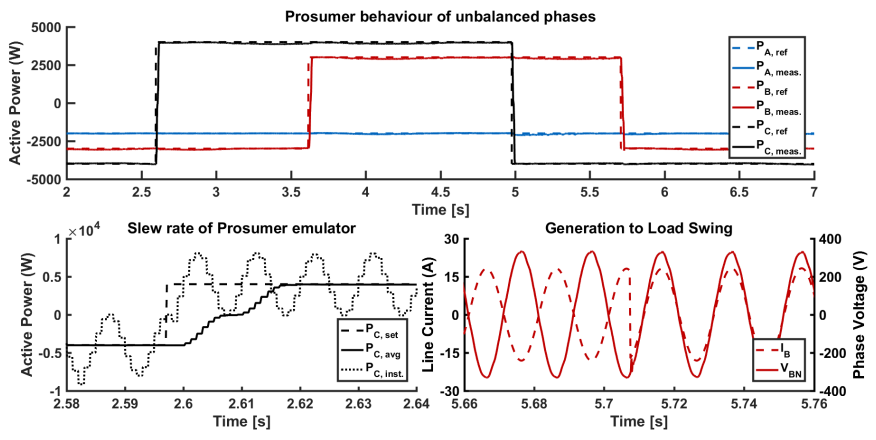

Fig. 5. PHIL validation results - prosumer operatation

with each other. Only the electricity price at the ends of the interconnecting node is available to both the clusters. The load CSUs receive their setpoints from a time series of an unbalanced three phase demand profile. The generator CSUs receive their setpoints from the local cluster control and OPF algorithm. The RT target at SFH3 and SFH4 control the power injections in Cluster \#1 and Cluster \#2 respectively. The RT target for the LV grid, measures power at the LV buses and provides PLL references to the Egston CSUs over the RMN network. Two separate Windows PC are used to deploy the VS projects, one each for RT target at SFH3 and SFH4, to represent the two clusters. The PCs run an ADMM OPF algorithm, split in two halves, and they communicate with each other over a TCP link. The algorithm receives the measured power at the load buses and directs the generation injection power after an optimisation step. We use JSONs to exchange the node prices over the TCP link. The VS LabVIEW API is used to transfer data across the static optimisation and RT emulation domains. Further explanation of the methodology can be found in [8].

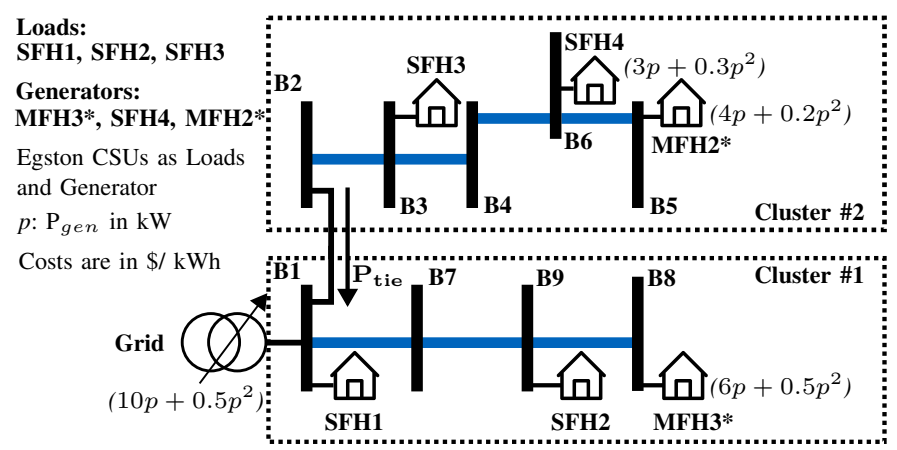

Fig. 6. PHIL experiment for OPF validation with two clusters. The generation costs are mentioned adjacent to the generators.

In the top row of Fig. 7, we show the comparison of generation mix of phase A for the two cases, Case \#1 with OPF and Case \#2 without OPF, for the same load profile. The experiment runs for $550 \mathrm{~s}$. The generator nodes are initially programmed to provide self-sufficiency for the local cluster as a primary control and keep the exchange of power between the two clusters, $\mathrm{P}_{t i e}$, at zero. In Case \#1, the MFH3* generator completely provides for Cluster \#1 while MFH2*
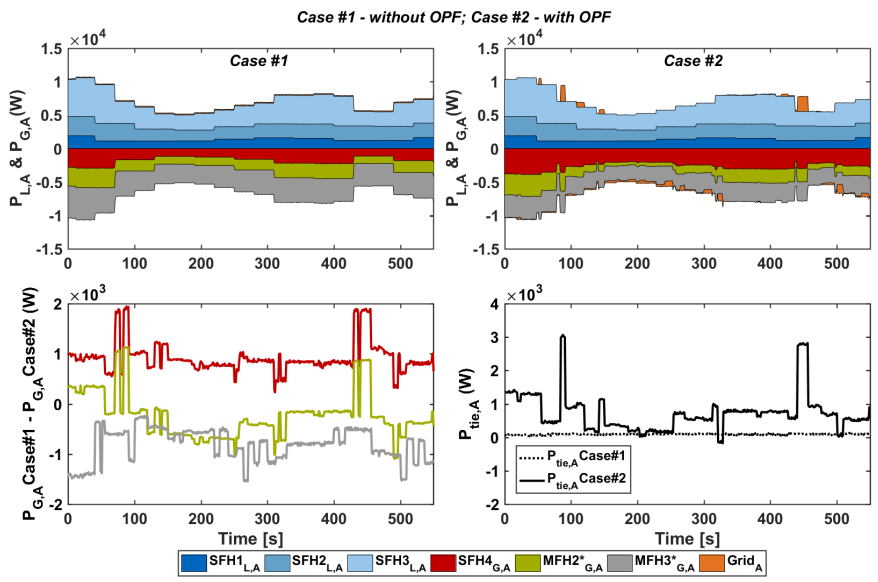

Fig. 7. Comparison of generation mix (top), change in $P_{g e n, A}$ (bottom left) and power exchanged between clusters (bottom right) with and without OPF

and SFH4 provide for Cluster \#2. Case \#2 redispatches the three generators to minimise the total cost through online OPF, which reduces the generation from Cluster \#1 and increases at Cluser \#2, as seen in the bottom left of Fig. 77. The bottom right of Fig. 7 shows the power sent from Cluster \#2 towards Cluster \#1 due to optimised generation costs in the OPF case. The total generation costs was $\$ 0.233$ for Case \#1 and $\$ 0.198$ for Case \#2, with an improvement of $\approx 15 \%$.

\section{CONClusion}

The CoSES microgrid laboratory was established to research microgrid operation through real and emulated multenergy system grids. In this paper, the electrical network and PHIL capacities of the lab were presented in detail. The design philosophies behind the design choices in the electrical and control system network of CoSES were explained. A schematic representation of the general PHIL experiment scheme in the lab was introduced. Validation results for the PHIL agents was shown in the results. One detailed microgrid experiment for distributed OPF validation was also shown for the reader's understanding of the lab potential in a research question context.

\section{REFERENCES}

[1] V. S. Perić, T. Hamacher, A. Mohapatra, F. Christiange, D. Zinsmeister, P. Tzscheutschler, and C. Aigner, "CoSES Laboratory for Combined Energy Systems At TU Munich," IEEE Power and Energy Society General Meeting, 2020.

[2] International Electrotechnical Commission, "IEC 60309-1:2021," Nov. 8, 2021 [Online].

[3] Egston Power, "COMPISO Power Hardware in the Loop," Nov. 8, 2021 [Online].

[4] NI, "Veristand 2018," Nov. 8, 2021 [Online].

[5] M. Mayer, A. Mohapatra, and V. S. Peric, "IoT Integration for Combined Energy Systems at the CoSES Laboratory," in 2021 IEEE 7th World Forum on Internet of Things (WF-IoT), pp. 195-200, 2021.

[6] LEM, "Lem transducers," Nov. 8, 2021 [Online].

[7] E. Sezgin, A. Mohapatra, V. S. Peric, O. Salor, and T. Hamacher, "Fast harmonic analysis for PHIL experiments with decentralized realtime controllers," submitted to PSCC 2022, http://dx.doi.org/10.36227/ techrxiv. 17061944

[8] M. Cornejo, A. Mohapatra, S. Candas, and V. S. Peric, "PHIL implementation of a decentralized online OPF for active distribution grids." submitted to PES GM 2022, http://dx.doi.org/10.36227/techrxiv.17065193 